\title{
Interaction of Protein-Bound Polysaccharide (PSK) with Smooth Muscle Myosin Regulatory Light Chain
}

\author{
Toshihiro FuJII ${ }^{*, a}$ and Mitoshi KunImatsu ${ }^{b}$ \\ ${ }^{a}$ Department of Kansei Engineering, Faculty of Textile Science and Technology, Shinshu University; Ueda, Nagano \\ 386-8567, Japan: and ${ }^{b}$ Department of Biochemistry, Nagoya City University Medical School; Kawasumi 1, Mizuho-cho, \\ Mizuho-ku, Nagoya 467-8601, Japan. Received December 19, 2002; accepted March 3, 2003
}

\begin{abstract}
The interaction of a protein-bound polysaccharide (PSK) isolated from Basidiomycetes with smooth muscle myosin components was evaluated by limited digestion, urea/glycerol gel electrophoresis, affinity chromatography and overlay assay using a peptide array. PSK was bound to the regulatory light chain (RLC) of myosin, but not to the essential light chain. The binding to PSK was definitely observed for unphosphorylated RLC, compared to phosphorylated one. From the amino acid sequence of the RLC, 490 peptides were synthesized on a cellulose membrane. Overlay assays showed that the PSK-binding on the molecule of RLC were localized in the N-and Cterminal basic regions and these sites were conserved in RLC from the human smooth muscle and nonmuscle cells.
\end{abstract}

Key words protein-bound polysaccharide; myosin; regulatory light chain; binding site; peptide array

The cytoskeletal proteins, myosin and actin, play an important role in the contraction of muscles and in a variety of cellular functions such as cell movement, shape change, and cytokinesis in nonmuscle cells. ${ }^{1,2)}$ In vertebrate smooth muscle and nonmuscle cells, the actin-activated $\mathrm{Mg}^{2+}$-ATPase activity of myosin, the myosin motor function activity, is regulated by phosphorylation of the $20 \mathrm{kDa}$ myosin regulatory light chain, which is catalyzed by $\mathrm{Ca}^{2+}$ - and calmodulin-dependent myosin light chain kinase (MLCK). ${ }^{3,4)}$

PSK, a protein-bound polysaccharide obtained from Basidiomycetes, has been shown to exhibit antitumor activity against a variety of tumor cells such as gastric, colorectal, and lung cancers. ${ }^{5,6}$ The antitumor effects of PSK have been also considered to be caused by its activity as an immunomodulator. The precise mechanism at the molecular level remains unknown. Previously, we found that PSK can significantly inhibit the actin-activated $\mathrm{Mg}^{2+}$-ATPase activity of myosin from rabbit skeletal muscle. ${ }^{7)}$ The marked ATPase inhibition is due to the interaction of PSK with myosin filaments, which induces side by side interactions between the myosin filaments to the long axis. ${ }^{8)}$ Kaneko and co-workers have reported that PSK causes an effective inhibition on the actinactivated $\mathrm{Mg}^{2+}$-ATPase activity of phosphorylated myosin from smooth muscle, though this drug enhances the MLCK activity. ${ }^{9)}$ They have speculated that the ATPase inhibition may be due to the PSK-binding to smooth muscle myosin in a similar way as in the case of its binding to skeletal muscle myosin. ${ }^{9,10)}$ However, there is no direct evidence for the interaction between PSK and smooth muscle myosin.

In this study, we report that PSK directly interacts with the regulatory light chain (RLC) of smooth muscle myosin in vitro. The interaction is clearly observed in unphosphorylated form of RLC. We also examined in detail the PSKbinding site on the molecule of RLC.

\section{MATERIALS AND METHODS}

Materials and Methods PSK and anti-PSK antibodies were purchased from Kureha Chemical Industries (Tokyo). The anti-phosphorylated RLC antibodies were a generous gift from Dr. Matsumura (Rutgers University). ${ }^{11)}$ Papain was purchased from Sigma. The CNBr-activated Sepharose was from Pharmacia. The peroxidase-conjugated anti-rabbit IgG was from Wako. All other chemicals were of the highest quality commercially available.

Preparations of Myosin, Myosin Light Chain Kinase and Calmodulin Myosin and myosin light chain kinase were purified from chicken gizzard smooth muscle according to Persechini and Hartshorne ${ }^{12)}$ and Ngai and Walsh, ${ }^{13)}$ respectively. Light chains from chicken gizzard myosin were prepared according to the method of Grand and Perry. ${ }^{14)}$ Calmodulin was isolated from porcine brain. ${ }^{15)}$

Phosphorylation of Myosin Myosin light chain phosphorylation was performed at $25^{\circ} \mathrm{C}$ for $30 \mathrm{~min}$ by adding $500 \mu \mathrm{g}$ of myosin to an assay medium $(400 \mu \mathrm{l})$ containing $20 \mathrm{~mm}$ Tris- $\mathrm{HCl}, \mathrm{pH}$ 7.0, $5 \mathrm{~mm} \mathrm{MgCl}_{2}, 2.5 \mathrm{~mm} \mathrm{CaCl}, 1 \mathrm{~mm}$ dithiothreitol (DTT), and $30 \mathrm{~mm} \mathrm{KCl}$ and $0.5 \mathrm{~mm}$ ATP with $11 \mu \mathrm{g}$ of chicken gizzard myosin light chain kinase and $10 \mu \mathrm{g}$ of calmodulin. The reaction was stopped by adding EDTA and then centrifuged at $12000 \times \boldsymbol{g}$ at $4{ }^{\circ} \mathrm{C}$ for $30 \mathrm{~min}$. The obtained pellets were dissolved in $10 \mathrm{~mm}$ Tris- $\mathrm{HCl}, \mathrm{pH}$ 7.5, $0.2 \mathrm{~mm}$ DTT and $300 \mathrm{~mm} \mathrm{KCl}$ and then used.

SDS-PAGE and Urea/Glycerol Electrophoresis Sodium dodecyl sulfate-polyacrylamide gel electrophoresis (SDSPAGE) was carried out on $12.5 \%$ polyacrylamide gel as described by Laemmli. ${ }^{16)}$ Urea/glycerol gel electrophoresis was performed according to the method of Sobieszek and Jertschin. ${ }^{17)}$ After electrophoresis, proteins in the gel were stained with $0.1 \%$ Coomassie Brilliant blue R-250, $10 \%$ acetic acid and $40 \%$ ethanol for $60 \mathrm{~min}$ and then destaining was carried out in $10 \%$ acetic acid and $40 \%$ ethanol.

Synthesis of Overlapping Peptides Peptide chips were prepared on derivatized cellulose membranes with 9-fluorenylmethoxycarbonyl amino acids according to the protocol from the manufacturer of the system (Auto spot robot ASP222; ABIMED Analysen-Technik GmbH, Langenfeld, Germany). ${ }^{18,19)}$ The ASP222 software program was used to generate the amino acid sequences of peptides with the 6,8 and 12 amino acid lengths and the spotting schedule for each cycle of addition of an amino acid. Peptides spanning amino 
acid residues $1-172$ of myosin light chain were synthesized on cellulose membranes as a series of peptides with one by one shifting.

Immunoblots and Overlay Assay After SDS-PAGE, the proteins in the gel were transferred onto a nitrocellulose membrane (Advantec). After blocking with 5\% BSA in TBS$\mathrm{T}$ (25 mm Tris- $\mathrm{HCl}, \mathrm{pH} 7.2,50 \mathrm{~mm} \mathrm{NaCl}, 0.5 \%$ Tween-20) and washing with TBS-T (4 times, for $10 \mathrm{~min}$ each), the membrane was incubated with anti-phosphorylated RLC specific antibodies. ${ }^{11)}$ Following extensive washes in TBS-T, the membrane was incubated with peroxidase-conjugated secondary antibodies against rabbit IgG (1/30000-fold dilution in TBS containing $1 \% \mathrm{BSA}$ ). The membrane was washed as above and the signals were detected by the enhanced chemiluminescence detection system (Super Signal CL-HRP Substrate System; Pierce). The detailed procedure of the overlay assay was described in the text.

Other Procedures PSK was coupled to CNBr-activated Sepharose 4B following the procedures outlined by Pharmacia. About $3 \mathrm{mg}$ of PSK was linked to $1 \mathrm{ml}$ of Sepharose. Myosin was digested with papain (1/200) in a solution containing $25 \mathrm{~mm}$ MES-KOH, pH 6.5, $2 \mathrm{~mm} \mathrm{MgCl}_{2}$, and $20 \mathrm{~mm}$ $\mathrm{KCl}$ at $25^{\circ} \mathrm{C}$ for $0-90 \mathrm{~min}$. The protein concentrations were determined by a Bio-rad Coomassie blue protein assay using BSA as the standard or by spectrophotometric measurements as previously described. ${ }^{15,18)}$

\section{RESULTS AND DISCUSSION}

Phosphorylation of Gizzard Myosin and the Effect of PSK on the Mobility of the Light Chains The molecule of smooth muscle myosin II is composed of two $200 \mathrm{kDa}$ heavy chains, two $20 \mathrm{kDa}$ RLC, and two $17 \mathrm{kDa}$ essential light chains (ELC). It is also known that the motor activity of smooth muscle myosin is activated by phosphorylation of regulatory light chain. ${ }^{1,2,21)}$ The phosphorylation level of the myosin light chain was determined by immunoblot using phosphorylated RLC specific polyclonal antibodies (specific for phosphorylated $\left.\mathrm{S}^{19}\right) .{ }^{1,21,22)}$ The immunoblot data showed that isolated chicken gizzard myosin contained little, if any, phosphorylated regulatory light chain, while the addition of calcium/calmodulin and MLCK to the myosin solution in the presence of ATP converted the regulatory light chain into the phosphorylated form (Fig. 1A). The mobility of RLC in urea/glycerol gel electrophoresis is different between the phosphorylated and unphosphorylated forms. ${ }^{11,17)}$ Figure 1B shows that most of the regulatory light chain was phosphorylated under our experimental conditions. Interestingly, the band of the unphosphorylated RLC disappeared with the increasing concentration of PSK added $(>0.5 \mathrm{mg} / \mathrm{ml})$, but the phosphorylated RLC and ELC did not, indicating the interaction between PSK and unphosphorylated RLC (Figs. 1B, C). Since the biochemical activities of smooth muscle and nonmuscle actomyosin system are dependent on the phosphorylation and unphosphorylation of RLC, the interaction of PSK with unphosphorylated RLC was investigated in detail.

Interaction of PSK with RLC We reported that the digestion of myosin heavy and light chains from skeletal muscle with trypsin and chymotrypsin was inhibited by the addition of PSK ${ }^{8)}$ Unphosphorylated smooth muscle myosin was digested with papain $(1 / 200 ; \mathrm{wt} / \mathrm{wt})$ in the absence and pres-
$\mathbf{A}$

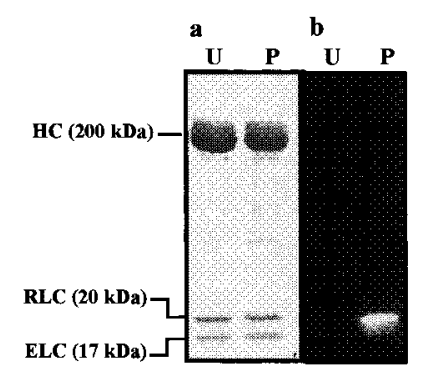

B

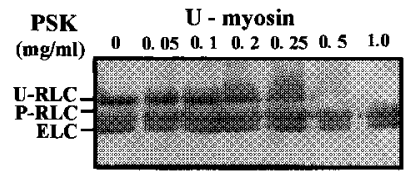

P - myosin

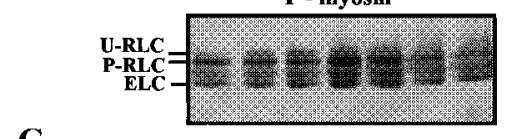

C

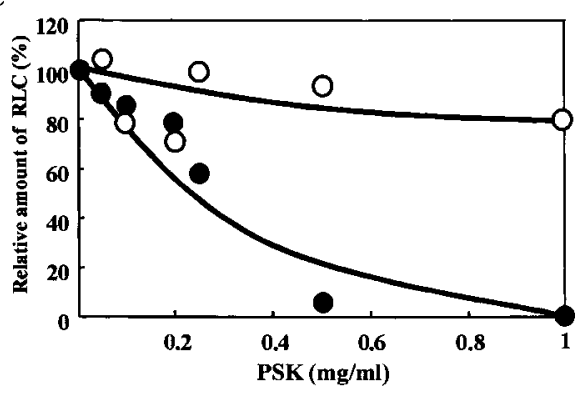

Fig. 1. Gel Electrophoresis of Unphosphorylated and Phosphorylated Chicken Gizzard Smooth Muscle Myosins and Their Interaction with PSK

Myosin was incubated with $\mathrm{Ca}^{2+} /$ calmodulin and MLCK and phosphorylated. The unphosphorylated $(\mathrm{U})$ and phosphorylated $(\mathrm{P})$ myosins were applied to $12.5 \%$ SDSPAGE and blotted onto a nitrocellulose membrane (A). a, Amide-black staining; b, antiphosphorylated RLC specific polyclonal antibodies. Bands of $200 \mathrm{kDa}$ heavy chain (HC), $20 \mathrm{kDa}$ RLC, and $17 \mathrm{kDa}$ ELC are indicated on the right. The unphosphorylated and phosphorylated myosins were incubated with $0-1 \mathrm{mg} / \mathrm{ml}$ PSK for $30 \mathrm{~min}$ at $30^{\circ} \mathrm{C}$ and applied to $14 \%$ urea/glycerol gel in which myosin heavy chains do not enter (B). The amounts of RLC were determined by densitometry (C). $\bullet$, Unphosphorylated RLC; O, phosphorylated RLC.

ence of $1.2 \mathrm{mg} / \mathrm{ml}$ PSK. Samples digested for various time periods at $25^{\circ} \mathrm{C}$ were analyzed by SDS-PAGE (Fig. 2). The major products were grouped around 140, 105, 75 and $30 \mathrm{kDa}$ fragments in the absence of PSK, while the product was a $140 \mathrm{kDa}$ fragment in the presence of PSK and production of the 105,75 and $30 \mathrm{kDa}$ fragments was virtually unobserved even after a 90-min digestion. Interestingly, RLC was not digested in the presence of PSK, but the digestion occurred over $20 \mathrm{~min}$ in the absence of PSK. Similar results, especially protection of RLC from digestion, were obtained when phosphorylated myosin was used in place of the unphosphorylated one. As previously reported, ${ }^{8)}$ PSK was not stained with Coomassie Brilliant Blue.

To ascertain the hypothesis that PSK directly binds to RLC, we prepared Light chains (LCs) from chicken gizzard myosin and applied them to a PSK-Sepharose column (Fig. 3). About half of the protein was retained on the column and the bound fraction was eluted at $400-450 \mathrm{~mm} \mathrm{NaCl}$. SDSPAGE showed that the unbound and bound fractions consisted of ELC and RLC, respectively. These results strongly suggest that PSK interacts with RLC. Furthermore, since urea $(7 \mathrm{M})$ was used for the preparation of the LCs to separate it from the myosin heavy chain, the interaction seemed to be 


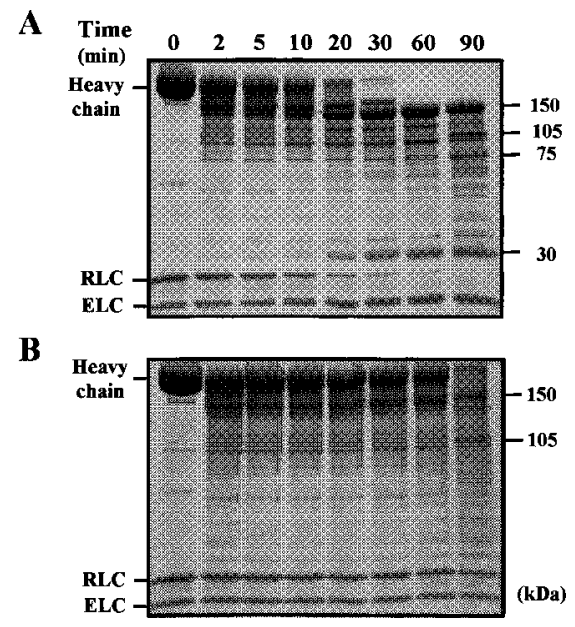

Fig. 2. Time Course of Myosin Digestion with Papain

Myosins $(0.3 \mathrm{mg} / \mathrm{ml})$ in the absence (A) and presence (B) of $1.2 \mathrm{mg} / \mathrm{ml}$ PSK were preincubated at $25^{\circ} \mathrm{C}$ for $20 \mathrm{~min}$ and further incubated for $90 \mathrm{~min}$ after the addition of $1.5 \mathrm{mg} / \mathrm{ml}$ papain. The samples after digestion were analyzed by $7.5-15 \%$ SDS-PAGE.

A

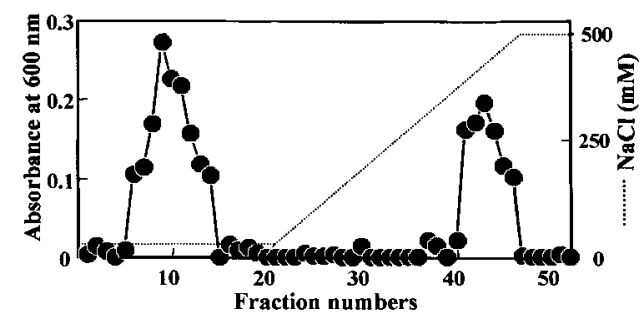

B

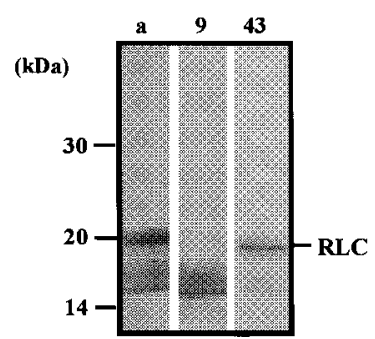

Fig. 3. PSK-Sepharose Affinity Chromatography of Smooth Muscle Light Chains (LCs)

The LCs $(1 \mathrm{mg})$ were loaded onto a PSK-Sepharose column $(2 \mathrm{ml})$ equilibrated with $20 \mathrm{~mm}$ MES-NaOH, pH 6.8, $30 \mathrm{~mm} \mathrm{NaCl}, 0.5 \mathrm{~mm} \mathrm{Mg}\left(\mathrm{CH}_{3} \mathrm{COO}\right)_{2}$, and $0.2 \mathrm{~mm}$ DTT. The column was washed with the above solution and the bound protein was eluted with a linear gradient of $30-500 \mathrm{~mm} \mathrm{NaCl}$ in the same buffer (---). Fractions were collected and analyzed for protein (- - The inset shows $12.5 \%$ SDS-PAGE for the applied sample (a), fractions 9 and 43 .

due to the amino acid sequence, not due to the steric conformation.

Identification of the PSK-Binding Sites of RLC Chicken gizzard smooth muscle RLC consists of 172 amino acid residues. From the amino acid sequence (Fig. 4), ${ }^{23}$ we synthesized peptide chips with 3 different amino acid lengths $(6,8,12)$ on cellulose membranes as spots (about $10 \mathrm{nmol}$ ). Peptides were synthesized by moving one by one, overlapping the peptide groups in the amino acid sequence without Met of the N-terminus. Four hundred ninety individual peptides were investigated for binding behavior to PSK using the overlay assay. The cellulose membrane was blocked with $5 \%$ BSA in TBS-T for at least $5 \mathrm{~h}$, washed with TBS-T, and incubated with $0.4 \mathrm{mg} / \mathrm{ml}$ PSK dissolved in TBS-T containing c. smooth muscle RLC:SSKRAKAKTTKKRPQRATSNVFAMFDQSQIOEFKEAFNMIDQN h. IYmphocyte RLC:SSKKAKTKTTKKRPQRATSNVFAMFDQSQIQEFKEAFNMIDQN : :

RDGFIDKEDLHDMLASMGKNPTDEYLEGMMSEAPGPINFTMFLTMFGEKLNGTDPEDVIRNAFA RDGFIDKEDLHDMLASLGKNPTDAYLDAMMNEAPGPINFTMFLTMFGEKLNGTDPEDVIRNAFA : : : : :

CFDEEASGFIHEDHTRELLTTMGDRFTDEEVDEMYREAPIDKKGNFNYVEFTRILKHGAKDKDD CFDEEATGTIQEDYLRELITTMGDRFTDEEVDELYREAPIDKKGNFNYIEFTRILKHGAKDKDD

$$
\text { : : : : : : }
$$

Fig. 4. Comparisons of Amino Acid Sequences of RLCs from Chicken Smooth Muscle and Human Lymphocyte

Colons show different amino acid sequences between them.
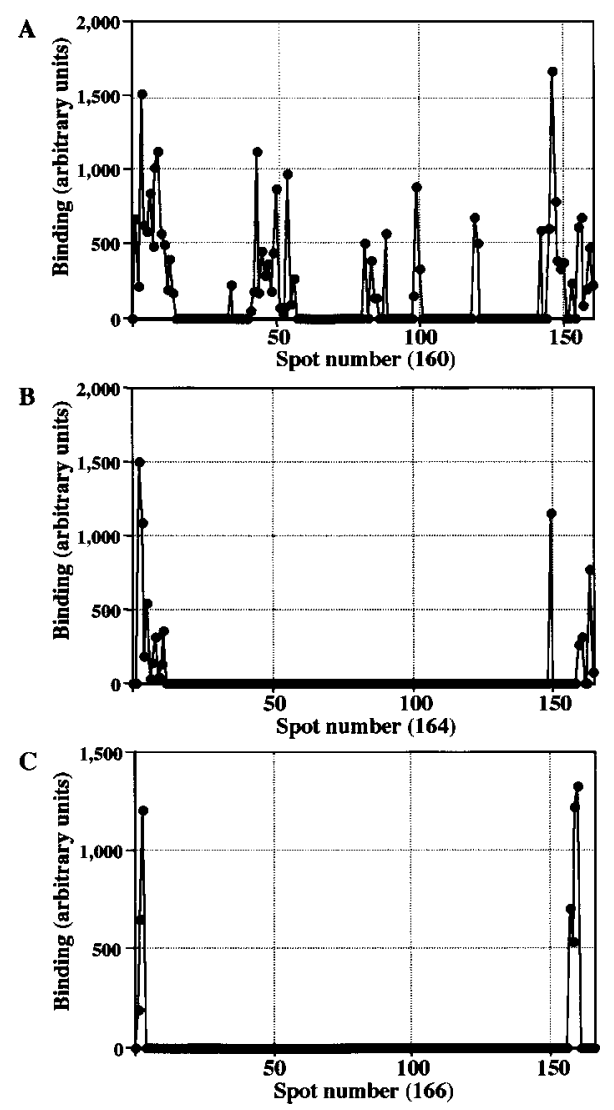

Fig. 5. Interaction of PSK with RLC using Peptide Array and Overlay Assay

The spot numbers represent the peptide synthesized on a cellulose membrane from the N-terminus of the amino acid sequence of chicken gizzard RLC. Bound PSK was detected as the chemiluminescent product. The light signal was determined by densitometric scanning and then plotted. A, Peptides with 12 amino acid length; B, peptides with 8 amino acid length; $C$, peptides with 6 amino acid length.

$1 \%$ BSA at $25^{\circ} \mathrm{C}$ for $60 \mathrm{~min}$. After washing, the membrane was incubated with $1: 1000$ dilution anti-PSK antibodies in TBS-T for $60 \mathrm{~min}$ at room temperature, washed with TBS-T and further incubated with the peroxidase-conjugated secondary antibodies against rabbit IgG for $60 \mathrm{~min}$ at room temperature. Following extensive washes in TBS-T, the membranes were visualized by the enhanced chemiluminescence detection system. ${ }^{24)}$ Figure 5 shows the binding of PSK to each peptide spot from the N-terminal amino acid sequence of RLC by densitometry. When the peptides with a 12 amino acid length were examined, there were at least 3-4 PSKbinding regions on the RLC molecule (Fig. 5A). On the other hand, when the amino acid lengths of peptides decreased to 6 and 8, the PSK bindings were localized at the $\mathrm{N}$ - and C-terminal regions (Figs. 5B and 5C). We speculated that the two 
sites of Ser1-Thr19 (N-site) and Glu157-Asp171 (C-site) seemed to be target for PSK-binding.

Interestingly, the basic amino acid content of the N-site $(8 / 19)$ and the basic and acidic amino acid contents (4/15 and $4 / 15$, respectively) of the $\mathrm{C}$-site were relatively high. No significant similarity between these amino acid sequences and those of ELC and heavy chain from chicken smooth muscle was found. It has been reported that MLCK could phosphorylate RLC at Thr18 and Ser19, and stimulate the actin-activated ATPase activity of myosin. ${ }^{1,11,20)}$ On the other hand, Kaneko et al. ${ }^{9}{ }^{\prime}$ reported that PSK inhibits the actin-activated ATPase activity of myosin, though the phosphorylation of myosin by MLCK is enhanced by PSK. These results suggest that the binding of PSK to the N-site will block the signal process after the phosphorylation of RLC. The amino acid sequences of the $\mathrm{N}$ - and $\mathrm{C}$-sites are very similar or the same between chicken smooth muscle and human lymphocytes (Nsite, 17/19; C-site, 15/15) (Fig. 4), ${ }^{23,25)}$ strongly indicating that PSK affects actomyosin system by interacting with RLC in human nonmuscle cells containing tumor cells.

PSK has been reported to suppress artificial metastasis models such as the metastasis of rat hepatoma AH60C, mouse colon cancer colon26, and mouse leukemia RL male 1 and spontaneous metastasis models. ${ }^{6}$ ) The metastasis is complicated and contained a number of processes including detachment of primary lesion, intravasation, intravascular migration, extravasation, migration after extravasation. Some of them are considered to be tightly associated with cell motility. These data strongly suggest that PSK exerts its biological activity through the binding to the myosin molecules.

Acknowledgments We wish to thank Dr. G. Taguchi (Gene Research Center, Shinshu University) for the helpful suggestions and discussions related to this work. This study was supported by Grants-in-Aid for COE Research (H10CE2003) and Science Research (C) (14593003) from the Ministry of Education, Culture, Sports, Science and Technology of Japan.

\section{REFERENCES}

1) Tan J. L., Ravid S., Spudich J. A., Annu. Rev. Biochem., 61, 721-759 (1992).

2) Mermall V., Post P. L., Mooseker M. S., Science, 279, 527-533 (1998).

3) Adelstein R. S., Eisenberg E., Annu. Rev. Biochem., 49, 921-956 (1980).

4) Somlyo A. P., Somlyo A. V., Nature (London), 372, 231-236 (1994).

5) Tsukagoshi S., Hashimoto Y., Fujii G., Kobayashi H., Nomoto K., Orita K., Cancer Treat. Rev., 11, 131-155 (1984).

6) Kobayashi H., Matsunaga K., Oguchi Y., Cancer Epidemiol. Biomarkers Prev., 4, 275-281 (1995).

7) Fujii T., Akabane Y., Tateishi Y., Fujii M., Kondo Y., Ohki K., Chem. Pharm. Bull., 33, 3408-3414 (1985).

8) Fujii T., Suzuki T., Akashi H., Hozumi T., Ogoma Y., Fujii M., Kondo Y., Biomed. Res., 8, 445-452 (1987).

9) Kaneko K., Takano-Ohmuro H., Iwai T., Kohama K., J. Biochem. (Tokyo), 110, 851-853 (1991).

10) Akashi H., Tezuka O., Ogoma Y., Kondo Y., Hozumi T., Fujii T., Biomed. Res., 9, 443-450 (1988).

11) Matsumura F., Ono S., Yamakita Y., Totsukawa G., Yamashiro S., J. Cell Biol., 140, 119-129 (1998).

12) Persechini A., Hartshorne D. J., Biochemistry, 22, 470-476 (1983).

13) Ngai P. K., Walsh M. P., J. Biol. Chem., 259, 13656-13659 (1984).

14) Grand R. J., Perry S. V., Biochem. J., 211, 267-272 (1983).

15) Fujii T., Oomatsuzawa A., Kuzumaki N., Kondo Y., J. Biochem. (Tokyo), 116, 121-127 (1994).

16) Laemmli U. K., Nature (London), 227, 680-685 (1970).

17) Sobieszek A., Jertschin P., Electrophoresis, 7, 417-425 (1986).

18) Fujiwara S., Takeo N., Otani Y., Parry D. A. D., Kunimatsu M., Lu R., Sasaki M., Matsuo N., Khaleduzzaman M., Yoshioka H., J. Biol. Chem., 276, 13340-13347 (2001).

19) Frank R., J. Immunol. Methods, 267, 13-26 (2002).

20) Bradford M. M., Anal. Biochem., 72, 248-254 (1976).

21) Kamm K. E., Stull J. T., Annu. Rev. Physiol., 51, 299-313 (1989).

22) Ikebe M., Hartshorne D. J., Elzinga M., J. Biol. Chem., 261, 36-39 (1986).

23) Messer N. G., Kendrick-Jones J., FEBS Lett., 234, 49-52 (1988).

24) Fujii T., Yabe S., Nakamura K., Koizumi Y., Biol. Pharm. Bull., 25, $573-579$ (2002)

25) Watanabe M., Kohri M., Takaishi M., Horie R., Higashihara M., J. Smooth Muscle Res., 37, 25-38 (2001). 